\title{
On abstract ovals with Pascalian secant lines
}

\author{
John Bamberg, Tim Harris, and Tim Penttila
}

\begin{abstract}
We prove that an abstract oval is an abstract conic if and only if all of its involutions are regular and all of its secant lines are Pascalian. In doing so, we provide a new characterisation of the rank 1 projective general linear groups over arbitrary fields.
\end{abstract}

\section{Introduction}

The theory of abstract ovals began with the three papers published in 1966 by Francis Buekenhout: Plans projectifs à ovoïdes pascaliens [7]; Ovales et ovales projectifs [6]; and Études intrinsèque des ovales [5]. Buekenhout realised that the "no three points collinear" property of an oval of a projective plane, and that "Pascal's theorem" for a conic, can be expressed entirely within the language of permutation groups. In so-doing, he created a link between multiply transitive groups and the geometry of projective planes. However, the true origin of the subject lies in Hilbert's Calculus of ends (1903) [17], with a line of descent that can be traced through Veblen \& Young (1910) [39], Bottema (1928) [4] and Coxeter (1949) [9].

Hilbert's calculus of ends can in turn be traced back to the discovery of the boundary at infinity of the hyperbolic plane by Beltrami (1868) [3] when he constructed the first models of non-Euclidean geometry. These ideal elements of geometry take their place alongside the points at infinity in projective geometry of Kepler (1604) [21] (influenced by the theory of perspective that grew out of Renaissance art), the point at infinity of inversive geometry of Möbius (1855) [31] (taken up by Riemann $(1851)^{1}$ and the Riemann sphere to study complex functions) and the circular points of Poncelet (1822) [32]. One remarkable result in Buekenhout's work is that if a Pascalian oval of a projective plane $\pi$ exists, then $\pi$ is Pappian and the oval is a conic [5, Proposition 7.9]. Another was an intrinsic study of ovals, leading to abstract ovals. In the ensuing years, there was great interest in extending Buekenhout's theorem on Pascalian ovals by many authors: Artzy (1968) [2], Rigby (1969) [34], Hofmann (1971) [18], Karzel \& Sörensen (1971) [19, 20] (with similar coordinates appearing in G. E. Martin (1971) [29]), Conti (1975) [8] and Korchmaros (1977/1978) [24, 25], until in 1980, Faina [12] offered an extension to abstract ovals, combining the concerns of the three papers.

Hofmann (1971) [18] proved that the five point Pascal property suffices to characterise a conic of a Pappian plane. Korchmaros $(1977 / 1978)[\mathbf{2 4 , 2 5}]$ studied the set of Pascal lines of an oval in a Desarguesian plane of even order, and Faina (1980) [12] proved

2010 Mathematics Subject Classification. 51A20, 51A30, 51E21, 20B22, 20E42.

Key words and phrases. abstract oval, Pascalian, Moufang set.

The first author acknowledges the support of the Australian Research Council Future Fellowship FT120100036.

${ }^{1}$ See B. Riemann, Grundlagen für eine allgemeine Theorie der Functionen einer vernderlichen complexen Grösse, Inaugural Dissertation (1851), Göttingen; pp.1-42 in [33]. 
his extension to abstract ovals. Korchmaros (1981) [26] showed that tangent lines and secant lines being Pascalian suffices. Amici \& Casciaro (1983) [1] showed that external Pascalian points suffice for projective planes of odd order. Fernandes (1984) [14] showed that a translation plane of order 3 modulo 4 which contains an oval with the four point Pascal property is a semifield plane and Fernandes (1993) [15] showed that the five point property on tangents and secants together with the four point property suffices.

Buekenhout [5] also proved that an abstract oval of odd order with all involutions having fixed points regular is a conic in a Pappian plane. Korchmaros (1975/1976) [23] proved the same theorem for even order ovals by using the Hering-Kantor-Seitz Theorem [16]. (Faina \& Korchmaros (1985) [13] gave a list of possibilities for the groups of 2transitive abstract ovals of even order, using deep theorems in group theory, but not the classification of the finite 2-transitive groups. Korchmaros (1987) [27] showed that the only 2-transitive abstract oval of order 3 modulo 4 is a conic, and proved a theorem that, after the classification of 2-transitive groups, also implies that result for order 1 modulo 4.)

Combining these two threads we prove a theorem extending the theorems of Buekenhout and of Korchmaros characterising conics as abstract ovals under conditions on regular involutions to the infinite setting by adding a hypothesis involving Pascalian lines:

TheOREM 1.1. An abstract oval $(\mathcal{O}, S)$ such that all the elements of $S$ are regular and all secant lines are Pascalian is a conic in a Pappian plane.

We prove this result in Theorem 3.17. Faina also assumed that all tangent lines are Pascalian. To be more precise, Faina showed that an infinite abstract oval is a conic in a Pappian plane if and only if it is Pascalian and one of two things occurs: (i) the identity belongs to $S$ and every nonidentity element of $S$ has a unique fixed point, or (ii) for every three distinct elements $A, B, C \in \mathcal{O}$ there is an involution $\kappa \in S$ such that $A^{\kappa}=B$, $C^{\kappa}=C, D^{\kappa}=D$, where $D=C^{\sigma}$ and $\sigma$ is the involution ${ }^{2}$ fixing $A$ and $B$. However, he had no hypothesis on regular involutions. Thus our theorem is a kind of blend of the theorems of Buekenhout and Korchmaros on finite abstract ovals and Faina's theorem: neither weaker nor stronger than either, as the finiteness hypothesis of Buekenhout and Korchmaros has been dropped (which was essential to their methods and enabled the utilisation of deep theorems on finite groups). We paraphrase Theorem 1.1 so that only the basic group theoretic notions are needed to understand the result.

Corollary 1.2. Let $G$ be a permutation group on $\mathcal{O}$, generated by a set $S$ of involutions of $G$ such that $t^{-1} s t \in S$ for all $s, t \in S$. Suppose that:

(i) for every $A_{1}, A_{2}, B_{1}, B_{2} \in \mathcal{O}$ with $\left\{A_{1}, A_{2}\right\} \cap\left\{B_{1}, B_{2}\right\}=\varnothing$ there is exactly one element $s$ of $S$ such that $A_{1}^{s}=A_{2}$ and $B_{1}^{s}=B_{2}$;

(ii) for all $A \neq B \in \mathcal{O}$ and all $s_{1}, s_{2}, s_{2} \in S$ taking $A$ to $B$, we have $s_{1} s_{2} s_{3} \in S$.

Then there is a field $\mathbb{F}$ such that the action of $G$ on $\mathcal{O}$ is permutationally isomorphic to the action of $\mathrm{PGL}(2, \mathbb{F})$ on $\mathrm{PG}(1, \mathbb{F})$. (Moreover, $\mathbb{F}$ has characteristic 2 only when $S$ also contains the identity element).

The proof of our theorem relies on two deep results: the first is by Segev [36] on special Moufang sets; the second is Tits' [37] characterisation of the projective general linear groups. (That the result uses Moufang sets is presaged by Korchmaros' [23] use of the Hering-Kantor-Seitz Theorem; the classification of finite Moufang sets.)

\footnotetext{
${ }^{2}$ Faina's axiom implies that every involution $\kappa \in S$ has at most two fixed points.
} 


\section{Abstract ovals}

An abstract oval $\mathcal{B}=(\mathcal{O}, S)$ is a set $\mathcal{O}$ of elements (where $|\mathcal{O}| \geqslant 3$ ) together with a set of involutory permutations $S$ such that for every $A_{1}, A_{2}, B_{1}, B_{2} \in \mathcal{O}$ with $\left\{A_{1}, A_{2}\right\} \cap\left\{B_{1}, B_{2}\right\}=\varnothing$ there is exactly one element $s$ of $S$ such that $A_{1}^{s}=A_{2}$ and $B_{1}^{s}=B_{2}$. Here we allow the identity permutation to be an involution, and by default, we will denote it by the symbol 1 . An abstract oval gives rise to an incidence structure $\mathbb{P}(\mathcal{B})$ as follows:

Points: the elements of $\mathcal{O}$ and the involutions $S$;

Lines: each line $\ell_{A, B}$ is defined by a pair of (not necessarily distinct elements) $A$, $B$ of $\mathcal{O}$, and is incident with $A, B$ and all elements of $S$ that map $A$ to $B$. (Such a line is secant if $A \neq B$ and tangent if $A=B$.)

An involution $s \in S$ is regular if it normalises $S$, and so $s$ induces an automorphism of $\mathbb{P}(\mathcal{B})$. A line $\ell$ of $\mathbb{P}(\mathcal{B})$ is said to be Pascalian if, for every three involutions $i, j, k \in \ell$, the product $i j k$ is also an involution in $S$ (in which case $i j k$ is also incident with $\ell$ ). An abstract oval is said to be Pascalian if every line of its associated incidence structure is Pascalian. A conic $\mathcal{O}$ of a Pappian projective plane $\Pi$ gives rise to a Pascalian abstract oval as follows. Given a point $P$ of $\Pi \backslash \mathcal{O}$ and a point $X$ of $\mathcal{O}$, we define $X^{P}$ to be the other point of $\mathcal{O}$ incident with $P X$ when $P X$ is a secant line, otherwise we set $X^{P}:=X$ if $P X$ is tangent to $\mathcal{O}$. Each point $P$ of $\Pi \backslash \mathcal{O}$ then gives rise to an involution $s_{P}: X \mapsto X^{P}$, and we let $S$ be the set of all such involutory permutations. Then $\mathcal{B}:=(\mathcal{O}, S)$ is an abstract oval known as an abstract conic. The incidence structure $\mathbb{P}(\mathcal{B})$, in this case, is isomorphic to the incidence structure we obtain from $\Pi$ by removing the lines that do not intersect $\mathcal{O}$ (i.e., the external lines). In general, if the incidence structure $\mathbb{P}(\mathcal{B})$ of an abstract oval $\mathcal{B}$ is isomorphic to the incidence structure arising from some abstract conic then we say that $\mathcal{B}$ is also an abstract conic.

The converse of Theorem 1.1 also holds:

THEOREM 2.1. An abstract conic has all involutions regular and all secant lines Pascalian.

Proof. Every Pappian plane is coordinatised by a field $\mathbb{F}$ and a (nonempty, nondegenerate) conic in that plane gives rise to an abstract oval isomorphic to (PG $(1, \mathbb{F}), S)$, where $S$ is the set of all involutions of $\operatorname{PGL}(2, \mathbb{F})$. (Moreover, $\mathbb{F}$ has characteristic 2 if and only if $S$ contains the identity element). Thus all involutions are regular. The stabiliser of a point pair $\{P, Q\}$ in $\operatorname{PGL}(2, \mathbb{F})$ is isomorphic to the semidirect product $K$ of the multiplicative group $\mathbb{F}^{*}$ of $\mathbb{F}$ and a cyclic group of order 2 inverting $\mathbb{F}^{*}$. Thus all elements of $K \backslash \mathbb{F}^{*}$ are involutions, and the product of any three of elements of $K \backslash \mathbb{F}^{*}$ is an element of $K \backslash \mathbb{F}^{*}$, and therefore an involution. Moreover, an element of $(\mathrm{PG}(1, \mathbb{F}), S)$ is incident with the secant line $P Q$ if and only if it interchanges $P$ and $Q$, that is, if and only if it is in $K \backslash \mathbb{F}^{*}$. Thus all secant lines are Pascalian.

\section{Axial elements and Moufang sets}

Throughout, we consider an abstract oval $\mathcal{B}:=(\mathcal{O}, S)$. Without Faina's axiom, we do not have at our disposal that every (nontrivial) element of $S$ fixes at most two elements. The next best thing is that a product of two distinct elements of $S$ fixes at most two elements, an observation we accredit to Buekenhout in [5].

Lemma 3.1. Let $(\mathcal{O}, S)$ be an abstract oval, and let $s$ and $t$ be distinct elements of $S$. Then st fixes at most two points. 
Proof. Suppose st fixes distinct points $A, B, C$ of $\mathcal{O}$. Then:

$$
A^{s}=A^{t}, \quad B^{s}=B^{t}, \quad C^{s}=C^{t} .
$$

Now by definition of an abstract oval, if $A^{s} \neq B$, then there is a unique element of $S$ mapping $A$ to $A^{s}$ and $B$ to $B^{s}$; but this would force $s$ to be equal to $t$. So we must have $A^{s}=B$. Therefore, by this argument, we have $A^{s}=B, B^{s}=C, C^{s}=A$; two of which yield a contradiction. (So for example, $A^{s}=B$ and $C^{s}=A$ yields $B=C$ ). Therefore, st does not fix three points.

Following in a similar vein to Rigby [34], we define a non-identity permutation $\alpha$ in the group $\langle S\rangle$ generated by $S$ to be axial if it has precisely two fixed points $X$ and $Y$, and for all distinct points $Z$ and $W$ different from $X$ and $Y$, the element of $S$ interchanging $Z$ and $W^{\alpha}$ and $W$ and $Z^{\alpha}$ fixes $\{X, Y\}$. The axis of $\alpha$ is its fixed-point set $\{X, Y\}$. From the definitions, an abstract oval $(\mathcal{O}, S)$ has 'Pascalian secant lines' if whenever three involutions $s_{1}, s_{2}, s_{3} \in S$ each map $X$ to $Y$, their product $s_{1} s_{2} s_{3}$ is an involution in $S$.

Lemma 3.2. Let $(\mathcal{O}, S)$ be an abstract oval such that all secant lines are Pascalian. Let $s$ and $t$ be elements of $S$ interchanging $X$ and $Y$. Then st is axial.

Proof. Let $\alpha=s t$. So $\alpha$ fixes $X$ and $Y$. Let $Z$ and $W$ be points other than $X, Y$, and let $u$ be the (unique) element of $S$ interchanging $Z$ and $W^{\alpha}$, and $W$ and $Z^{\alpha}$. Now there is a unique element $r$ of $S$ such that $Z^{r}=W^{\alpha}$ and $X^{r}=Y$. Since each secant line is Pascalian, str is an involution in $S$. In particular, str $=(s t r)^{-1}=r t s$ and hence $W^{r t s}=W^{s t r}=Z$; that is, $W^{r}=Z^{\alpha}$. By uniqueness, $u=r$ and hence $u$ maps $X$ to $Y$. Therefore, st is axial.

Lemma 3.3. Let $(\mathcal{O}, S)$ be an abstract oval such that all secant lines are Pascalian. Let $\alpha$ be an axial element with axis $\{X, Y\}$ and let $s$ be an element of $S$ mapping $X$ to $Y$. Then there exists an involution $t \in S$ interchanging $X$ and $Y$ such that $\alpha=$ st.

Proof. Let $Z$ be a point not equal to $X$ or $Y$. Since an axial element fixes precisely two points, we cannot have $Z^{\alpha} \in\{X, Y\}$, and similarly, $Z^{s} \notin\{X, Y\}$ as $Z \notin\{X, Y\}$. Therefore, $\{X, Y\} \cap\left\{Z^{s}, Z^{\alpha}\right\}=\varnothing$. By definition of an abstract oval, there is a unique element $t \in S$ such that $X^{t}=Y$ and $\left(Z^{s}\right)^{t}=Z^{\alpha}$. Consider an arbitrary element $L$ of $\mathcal{O}$. If $L \in\{X, Y\}$, then $L^{\alpha}=L=L^{s t}$. So suppose $L \notin\{X, Y\}$. By definition of an axial map, the unique element $u$ interchanging $L$ and $Z^{\alpha}$, and $Z$ and $L^{\alpha}$, fixes $\{X, Y\}$. Since all secant lines are Pascalian, the product tsu is an involution of $S$ fixing $\{X, Y\}$, and in particular, $t s u=(t s u)^{-1}=$ ust. So

$$
\left(L^{\alpha}\right)^{t s}=L^{\alpha(t s u) u}=L^{\alpha(u s t) u}=Z^{s t u}=\left(Z^{s}\right)^{t u}=Z^{\alpha u}=L
$$

for all $L \in \mathcal{O}$, and therefore, $\alpha=s t$.

Lemma 3.4. Let $(\mathcal{O}, S)$ be an abstract oval such that all secant lines are Pascalian, and let $X$ and $Y$ be distinct elements of $\mathcal{O}$. Then, together with the identity transformation, the axial maps with a common axis $\{X, Y\}$ form an Abelian group $A(X, Y)$.

Proof. Let $\alpha$ and $\alpha^{\prime}$ be two axial elements with axis $\{X, Y\}$. By Lemma 3.3, there exist elements $p, q, p^{\prime} \in S$ such that $\alpha=p q$ and $\alpha^{\prime}=q p^{\prime}$. Therefore,

$$
\alpha \alpha^{\prime}=p q q p^{\prime}=p p^{\prime}
$$

and so by Lemma 3.2, $\alpha \alpha^{\prime}$ is axial with axis $\{X, Y\}$. Moreover, $\alpha^{-1}=(p q)^{-1}=q p$, and so again by Lemma 3.2, we see that the inverse of an axial map is axial (with the same axis). Therefore, together with the identity transformation, the axial maps with a 
common axis $\{X, Y\}$ form a group $A(X, Y)$. Next, since every secant line is Pascalian, $p^{\prime} p q$ is an involution and therefore,

$$
p^{\prime} p q=\left(p^{\prime} p q\right)^{-1}=q p p^{\prime}
$$

and hence

$$
\alpha \alpha^{\prime}=p p^{\prime}=q\left(q p p^{\prime}\right)=q\left(p^{\prime} p q\right)=\alpha^{\prime} \alpha .
$$

So $\alpha$ and $\alpha^{\prime}$ commute. This shows that $A(X, Y)$ is Abelian.

Lemma 3.5. Let $(\mathcal{O}, S)$ be an abstract oval such that all secant lines are Pascalian. Let $W, X, Y, Z$ be distinct elements of $\mathcal{O}$. Then there is a unique axial element $\alpha$ with axis $\{X, Y\}$ mapping $Z$ to $W$.

Proof. Let $s \in S$ be such that $s$ fixes $\{X, Y\}$. Then by definition of an abstract oval there is an element $t \in S$, fixing $\{X, Y\}$ such that $t$ maps $Z^{s}$ to $W$. Let $\alpha:=s t$. Then by Lemma 3.2, $\alpha$ is axial and maps $Z$ to $W$. Suppose $\alpha^{\prime}$ is another such axial element mapping $Z$ to $W$. Then by Lemma 3.3, we can write $\alpha^{\prime}=s t^{\prime}$ for some $t^{\prime} \in S$. But then $Z^{\alpha^{\prime}}=W$ implies that $\left(Z^{s}\right)^{t^{\prime}}=W$, and hence $t=t^{\prime}$ (by definition of an abstract oval). Therefore, $\alpha^{\prime}=\alpha$ and we have established the uniqueness of an axial element (with axis $\{X, Y\})$ mapping $Z$ to $W$.

Lemma 3.6. Let $(\mathcal{O}, S)$ be an abstract oval such that every element of $S$ is regular and all secant lines are Pascalian. Let $H$ be the group generated by $S$, and let $M$ be the stabiliser of a point $X \in \mathcal{O}$ in $H$. Then for each $Y \in \mathcal{O}$, the group of axial elements $A(X, Y)$ with axis $\{X, Y\}$ is a normal subgroup of $M_{Y}$.

Proof. Let $\alpha$ be an axial element with axis $\{X, Y\}$ and let $h \in H$. By Lemma 3.3, we can write $\alpha=s t$ for some $s, t \in S$. Since every element of $S$ is regular, $h^{-1} s h, h^{-1} t h \in S$. Now $h^{-1} \alpha h$ is a product of these two elements of $S$, and $X^{h}$ and $Y^{h}$ are both fixed by $h^{-1} \alpha h$. So it follows from Lemma 3.2 that $h^{-1} \alpha h$ is an axial element with axis $\left\{X^{h}, Y^{h}\right\}$. If furthermore, $h$ stabilises $\{X, Y\}$, then $h^{-1} \alpha h \in A(X, Y)$. In particular, $h^{-1} \alpha h \in A(X, Y)$ for all $h \in M_{Y}$.

Jacques Tits [38] introduced the concept of a Moufang set as an abstraction of certain rank 1 doubly transitive permutation groups. They are precisely the Moufang buildings of rank one. A Moufang set is a set $M$ with at least 3 elements together with a collection of subgroups $\left\{U_{m}: m \in M\right\}$ of permutations of $M$ satisfying the following two properties:

1. For each $m \in M, U_{m}$ fixes $m$ and acts regularly on $M \backslash\{m\}$.

2. For all $m \in M, U_{m}$ permutes the set $\left\{U_{y}: y \in M\right\}$ by conjugation.

The group $\Gamma$ generated by the so-called root subgroups $\left\{U_{m}: m \in M\right\}$ acts 2transitively on $M$ and is called the little projective group of $M$. We can think of a Moufang set simply as a 2-transitive permutation group whose point stabiliser contains a normal subgroup which is regular on the remaining points.

THEOREM 3.7. Let $(\mathcal{O}, S)$ be an abstract oval such that every element of $S$ is regular and all secant lines are Pascalian. Let $H$ be the group generated by $S$ and let $X \in \mathcal{O}$. Then $\mathcal{O} \backslash\{X\}$ together with the subgroups $\{A(X, Y): Y \in \mathcal{O} \backslash\{X\}\}$ form a Moufang set with Abelian root subgroups.

Proof. By definition of an abstract oval $(\mathcal{O}, S)$, the group $H$ generated by $S$ acts 3 transitively on $\mathcal{O}$ (see also Proposition 2.2 of [5]). Therefore, the point stabiliser $H_{X}$ acts 2-transitively on $\mathcal{O} \backslash\{X\}$. Let $Y$ be a point apart from $X$, and consider the stabiliser $H_{X, Y}$ of both $X$ and $Y$. By Lemma 3.6, $A(X, Y)$ is a normal subgroup of $H_{X, Y}$. Furthermore, by Lemma 3.4, $A(X, Y)$ is Abelian, and by Lemma 3.5, $A(X, Y)$ acts regularly on $\mathcal{O} \backslash\{X, Y\}$. 
Therefore we have a Moufang set on $\mathcal{O} \backslash\{X\}$ with Abelian root subgroups $\{A(X, Y)$ : $Y \in \mathcal{O} \backslash\{X\}\}$.

There is a way to construct every Moufang set from a group $U$ and a permutation $\tau$ of $U^{*}$, which we denote by $\mathbb{M}(U, \tau)$, and we describe it as follows (see also [10, $\left.\S 3\right]$ ). Let $X$ be the disjoint union of $U$ and $\{\infty\}$, where $\infty$ is a new symbol, and let 0 be the identity element of $U$. For each $u \in U$, define $\alpha_{u}$ to be the permutation of $X$ that right multiplies the elements of $U$ by $u$ and fixes $\infty$. Let $U_{\infty}:=\left\{\alpha_{u}: u \in U\right\}$ and extend $\tau$ to $\operatorname{Sym}(X)$ by setting $0^{\tau}=\infty$ and $\infty^{\tau}=0$. We then define $U_{0}:=U_{\infty}^{\tau}$ and $U_{u}:=U_{0}^{\alpha_{u}}$, for every $u \in U$. Finally, we let $\mathbb{M}(U, \tau)$ consist of $X$ together with the root subgroups $\left\{U_{x}: x \in X\right\}$. A necessary and sufficient condition for $\mathbb{M}(U, \tau)$ to be a Moufang set was provided by De Medts and Weiss [11], and it is essentially that every Hua map to $U$ is an automorphism of $U$.

In the proof of Theorem 3.12 (below), we need to identify the Moufang set $M$ of Theorem 3.7 with a Moufang set of the form $\mathbb{M}(U, \tau)$ in order to apply the definition of special. Hence we provide the following 'folklore' result.

Lemma 3.8. Let $G$ be a 2-transitive group on $\Omega$ with $0 \in \Omega$ and $\infty \in \Omega$, and let $U$ be a normal subgroup of the stabiliser of $\infty$ in $G$, with $U$ acting regularly on $\Omega \backslash\{\infty\}$. Then for any element $\tau$ of $G$ interchanging 0 and $\infty$, we have that $\mathbb{M}(U, \tau)$ is a Moufang set with little projective group $\left\langle U, U^{\tau}\right\rangle$.

Proof. It is sufficient to show that $G_{1}:=\left\langle U, U^{\tau}\right\rangle$ is 2-transitive on $\Omega$ and that $U$ is a normal subgroup of the stabiliser of $\infty$ in $G_{1}$, regular on $\Omega \backslash\{\infty\}$. Now $\tau$ moves $\infty$, so $G_{1}$ is transitive on $\Omega$ (since the orbits of $U$ on $\Omega$ are $\{\infty\}$ and $\Omega \backslash\{\infty\}$ ), and the stabiliser of $\infty$ in $G_{1}$ contains $U$, so $G_{1}$ is 2-transitive on $\Omega$. Moreover, the normality of $U$ in $G_{\infty}$ implies the normality of $U$ in the stabiliser of $\infty$ in $G_{1}$. By hypothesis, $U$ acts regularly on $\Omega \backslash\{\infty\}$, and so it follows that $\mathbb{M}(U, \tau)$ is a Moufang set. Now consider the little projective group $\Gamma$, which is generated by the root subgroups: $\{U\} \cup\left\{U^{\tau u}: u \in U\right\}$. Now if $x, u \in U$, then $x^{\tau u}=u^{-1} x^{\tau} u \in U U^{\tau} U$. Therefore, $U^{\tau u} \leqslant\left\langle U, U^{\tau}\right\rangle$ and so $G_{1}$ contains all of the root subgroups. Hence, $G_{1}=\Gamma$.

A Moufang set $\mathbb{M}(U, \tau)$ is said to be special if the following identity holds:

$$
\left(u^{-1}\right)^{\tau}=\left(u^{\tau}\right)^{-1}, \quad \text { for all } u \in U^{*} .
$$

So, in light of Lemma 3.8, if $G$ is a 2-transitive group on $\Omega$ with $0 \in \Omega$ and $\infty \in \Omega, U$ is a normal subgroup $G_{\infty}$ with $U$ acting regularly on $\Omega \backslash\{\infty\}$, and $\tau \in G$ interchanges 0 and $\infty$, then $\mathbb{M}(U, \tau)$ is special if and only if

$$
\left(0^{\alpha_{\omega}^{-1}}\right)^{\tau}=0^{\alpha_{\omega^{\tau}}^{-1}}, \quad \text { for all } \omega \in \Omega \backslash\{0, \infty\}
$$

where $\alpha_{\omega}$ is the unique element of $U$ mapping 0 to $\omega$ (and similarly, $\alpha_{\omega^{\tau}}$ is the unique element of $U$ mapping 0 to $\omega^{\tau}$ ).

EXAMPLE 3.9. Let $\mathbb{F}$ be a field of cardinality at least 3 , and consider the projective general linear group $G:=\operatorname{PGL}(2, \mathbb{F})$ acting naturally as Möbius transformations of the projective line $\mathrm{PG}(1, \mathbb{F}) \cong \mathbb{F} \cup\{\infty\}$. The Möbius transformation that maps each $z \in$ $\mathbb{F} \cup\{\infty\}$ to $\frac{a z+b}{c z+d}$ will be denoted $f_{a, b ; c, d}$. Note that the stabiliser $G_{\infty}$ of $\infty$ consists of the affine transformations $\left\{f_{a, b ; 0,1} \mid a, b \in \mathbb{F}, a \neq 0\right\}$. Let $U$ be the subgroup of translations: $\left\{f_{1, b ; 0,1} \mid b \in \mathbb{F}\right\}$. Notice that $U$ is a normal subgroup of $G_{\infty}$, and it acts regularly on $\mathrm{PG}(1, \mathbb{F}) \backslash\{\infty\}$. Now take the involution $\tau:=f_{0,-1 ; 1,0}$ (i.e., the map $z \mapsto-1 / z$ ). Note that $\tau$ interchanges 0 and $\infty$. Let $\omega$ be an element of $P G(1, \mathbb{F}) \backslash\{\infty\}$, and let $\alpha_{\omega}$ be the unique 
element of $U$ mapping 0 to $\omega$; that is, $\alpha_{\omega}$ is the translation $f_{1, \omega ; 0,1}$. So $\alpha_{\omega}^{-1}=f_{1,-\omega ; 0,1}$ and

$$
\left(0^{\alpha_{\omega}^{-1}}\right)^{\tau}=\left(0^{f_{1,-\omega ; 0,1}}\right)^{\tau}=(-\omega)^{\tau}=\frac{1}{\omega}=0^{f_{1,1 / \omega ; 0,1}}=0^{\alpha_{-1 / \omega}^{-1}}=0^{\alpha_{\omega \tau}^{-1}} .
$$

So we see that we have a special Moufang set on $\mathrm{PG}(1, \mathbb{F})$.

ExAmple 3.10. Now consider the affine general linear group $G:=\operatorname{AGL}(1, \mathbb{F})$ acting sharply 2-transitively as affine transformations of $\mathbb{F}$. Note that the stabiliser $G_{1}$ of $1 \in \mathbb{F}$ consists of the affine transformations $\{x \mapsto a x+1-a \mid a \in \mathbb{F} \backslash\{0\}\}$. Let $U:=G_{1}$. Notice that $U$ is trivially a normal subgroup of $G_{\infty}$, and it acts regularly on $\mathbb{F} \backslash\{1\}$. Now take the involution $\tau: x \mapsto-x+1$. Note that $\tau$ interchanges 0 and 1 . Let $\omega$ be an element of $\mathbb{F} \backslash\{0,1\}$, and let $\alpha_{\omega}$ be the unique element of $U$ mapping 0 to $\omega$; that is, $\alpha_{\omega}$ is the map $x \mapsto(1-\omega) x+\omega$. So $\alpha_{\omega}^{-1}$ is the map $x \mapsto \frac{1}{1-\omega}(x-\omega)$ and

$$
\begin{aligned}
\left(0^{\alpha_{\omega}^{-1}}\right)^{\tau} & =\left(\frac{-\omega}{1-\omega}\right)^{\tau}=\frac{\omega}{1-\omega}+1 . \\
0^{\alpha^{\top} \tau} & =\frac{-\omega^{\tau}}{1-\omega^{\tau}}=\frac{\omega-1}{\omega} .
\end{aligned}
$$

These two quantities are equal only when $\omega^{2}-\omega+1=0$. So, as long as $\mathbb{F}$ has cardinality greater than 4, we see that we have a Moufang set here that is not special.

We will need the following very important result in the theory of Moufang sets with Abelian root groups.

Theorem 3.11 (Segev 2009, [36]). Let $M$ be a Moufang set having Abelian root subgroups. If $M$ is not special, then the little projective group of $M$ is sharply 2-transitive.

The converse of the above result is still a wide open problem in the theory of Moufang sets. That is, it is not yet known that if $M$ is special then it has Abelian root subgroups.

THEOREM 3.12. If the ground set $\mathcal{O}$ has cardinality greater than 5 , then the Moufang set $\mathbb{M}(U, \tau)$ arising from Theorem 3.7 is not special.

Proof. Let $Y$ and $Z$ be distinct points not equal to $X$, and let $\tau$ be the unique involution fixing $X$ and mapping $Y$ to $Z$. Let $U$ be the group $A(X, Y)$ generated by the axial maps with axis $\{X, Y\}$. So from Lemma 3.8 and Theorem 3.7, $\mathbb{M}(U, \tau)$ is a Moufang set. Suppose $\mathbb{M}(U, \tau)$ is a special Moufang set. We point out that in this proof, $Y$ plays the role of ' $\infty$ ' and $Z$ plays the role of ' 0 ' in the definition of $\mathbb{M}(U, \tau)$. Let $W \in \mathcal{O} \backslash\{X, Y, Z\}$, let $\alpha_{W}$ be the unique axial map of $A(X, Y)$ mapping $Z$ to $W$, and let $\alpha_{W^{\tau}}$ be the unique axial map of $A(X, Y)$ mapping $Z$ to $W^{\tau}$. By Equation (1), we have

$$
\left(Z^{\alpha_{W}^{-1}}\right)^{\tau}=Z^{\alpha_{W \tau}^{-1}}
$$

Let $q$ be the unique involution that fixes $Z$ and interchanges $X$ and $Y$. By Lemma 3.3, there exist elements $s$ and $p$ of $S$ (interchanging $X$ and $Y$ ) such that $\alpha_{W}=q s$ and $\alpha_{W^{\tau}}^{-1}=q p$. Therefore, Equation (2) becomes

$$
Z^{s q \tau}=Z^{q p}
$$

and we also have

$$
Z^{q s \tau q p}=Z
$$

as $Z^{q s}=W$ and $W^{\tau q p}=Z$ (by definition of $\alpha_{W}$ and $\alpha_{W^{\tau}}^{-1}$ ). Since $q$ fixes $Z$, we can rewrite these two equations as

$$
Z^{s q \tau}=Z^{p}=Z^{s \tau q}
$$


Hence, if $Z_{0}:=Z^{s}$, then

$$
Z_{0}^{q q^{\tau}}=Z^{s q \tau q \tau}=Z^{s \tau q q \tau}=Z^{s}=Z_{0}
$$

Note that $q^{\tau} \in S$ as every involution of $S$ is regular. Now the inverse of $q q^{\tau}$ is $q^{\tau} q$, which must also fix $Z_{0}$. Therefore,

$$
\left(Z_{0}^{q}\right)^{q q^{\tau}}=Z_{0}^{q^{\tau}}=Z_{0}^{\left(q^{\tau} q\right) q}=Z_{0}^{q}
$$

So $q q^{\tau}$ fixes $Z_{0}$ and $Z_{0}^{q}$. By Lemma 3.1, $q q^{\tau}$ fixes at most two points. Therefore, there are at most two possibilities for $s$ because elements of $S$ that interchange $X$ and $Y$ are defined by their image of $Z$. So we have shown that there are at most two nontrivial elements of $A(X, Y)$, which is a contradiction if the ground set $\mathcal{O}$ of the abstract oval (in Theorem 3.7) has more than 5 elements. Therefore, $\mathbb{M}(U, \tau)$ is not special.

REMARK 3.13. We remark that in the case that $S$ is the set of involutions of $\operatorname{PGL}(2,4)$ (acting naturally on five points), the axial elements fixing two points $X$ and $Y$ form a subgroup $U$ of order 3 , and it turns out that the Moufang set $\mathbb{M}(U, \tau)$ is special in this case.

Corollary 3.14. Let $(\mathcal{O}, S)$ be an abstract oval such that all involutions are regular and all secant lines are Pascalian, and let $\Gamma$ be the group generated by the axial elements that fix a point $X \in \mathcal{O}$. Then $\Gamma$ is sharply 2 -transitive and isomorphic to $\operatorname{AGL}(1, \mathbb{F})$, for some field $\mathbb{F}$, acting naturally on the affine line $\mathrm{AG}(1, \mathbb{F})$.

Proof. First we consider the case that $\mathcal{O}$ has cardinality at most 5 . We refer to [?] where it is ascertained that $(\mathcal{O}, S)$ is an abstract conic in this case.

So suppose $\mathcal{O}$ has cardinality greater than 5. By Theorem 3.11, Theorem 3.7 and Theorem 3.12, $H_{X}$ gives rise to a non-special Moufang set with Abelian root subgroups $\{A(X, Y): Y \in \mathcal{O} \backslash\{X\}\}$, and so the little projective group $\Gamma$ is sharply 2-transitive (on $\mathcal{O} \backslash\{X\})$. Moreover, if $Y \in \mathcal{O} \backslash\{X\}$, then $\Gamma_{Y}=A(X, Y)$ by [11, Theorem 3.1(ii)]. Since $\Gamma_{Y}$ is Abelian, by the Kerby-Wefelscheid/Mazurov Theorem (see [22, Corollary 3] and $[30])$, the action of $\Gamma$ on $\mathcal{O} \backslash\{X\}$ is permutationally isomorphic to $\operatorname{AGL}(1, \mathbb{F})$ acting on $\mathbb{F}$, for some field $\mathbb{F}$.

We will need the following two results: the first of which is a well-known result in permutation group theory; the second is a consequence of [5, Proposition 2.4], which is in turn a consequence of Tits' characterisation of the projective general linear groups $[\mathbf{3 7}$, II. B., page 42] .

Theorem 3.15 (c.f., [28, pp. 41-42] and [35, §10.6.16]). Let $G \leqslant \operatorname{Sym}(\Delta)$ be $k$ transitive, $k>1$. Extend $\Delta$ by an element $\infty$ and suppose $y$ is an involutary permutation of $\Delta \cup\{\infty\}$ that does not fix $\infty$. If there exists an involution $x \in G$, moving a point $\delta \in \Delta$ such that $(x y)^{3}=1$ and $G_{\delta}^{y}=G_{\delta}$, then $\hat{G}:=\langle G, y\rangle$ acts $(k+1)$-transitively on $\Delta \cup\{\infty\}$ with $\hat{G}_{\infty}=G$.

THEOREM 3.16. Let $(\mathcal{O}, S)$ be an abstract oval. Then $(\mathcal{O}, S)$ is an abstract conic if and only if the group $H$ generated by $S$ is sharply 3-transitive on $\mathcal{O}$.

We now have our main result:

TheOREM 3.17. An abstract oval $(\mathcal{O}, S)$ such that every element of $S$ is regular and all secant lines are Pascalian is an abstract conic.

Proof. Let $H=\langle S\rangle$, and let $X \in \mathcal{O}$. By Theorem 3.7, $\mathcal{O} \backslash\{X\}$ together with the subgroups $\{A(X, Y): Y \in \mathcal{O} \backslash\{X\}\}$ form a Moufang set with Abelian root subgroups. Let $\Gamma$ be the little projective group of this Moufang set (and so $\Gamma \leqslant H_{X}$ ). By Corollary 
3.14, $\Gamma \cong \operatorname{AGL}(1, \mathbb{F})$ for some field $\mathbb{F}$, and hence $\Gamma$ has a non-identity involution $x$. (To see this, $t \mapsto-t$ is an involution when the characteristic of $\mathbb{F}$ is not 2 , whereas, $t \mapsto t+1$ is an involution when the characteristic of $\mathbb{F}$ is 2). So let $Y$ be an element of $\mathcal{O} \backslash\{X\}$ that is moved by $x$ (i.e., $Y^{x} \neq Y$ ). Let $Z:=Y^{x}$. Note that we must have $x \in S$, as $\Gamma$ is sharply 2-transitive on $\mathcal{O} \backslash\{X\}$. Let $y$ be the unique involution in $S$ that fixes $Y$ and interchanges $X$ and $Z$. Let $H^{\prime}:=\langle\Gamma, y\rangle$. Then

$$
\begin{aligned}
& X^{x y x y x y}=X^{y x y x y}=Z^{x y x y}=Y^{y x y}=Y^{x y}=Z^{y}=X, \\
& Y^{x y x y x y}=Z^{y x y x y}=X^{x y x y}=X^{y x y}=Z^{x y}=Y^{y}=Y, \\
& Z^{x y x y x y}=Y^{y x y x y}=Y^{x y x y}=Z^{y x y}=X^{x y}=X^{y}=Z .
\end{aligned}
$$

So $(x y)^{3}$ fixes three points. However, $(x y)^{3}=y^{x} x^{y}$, which is a product of two elements of $S$ (as $x$ and $y$ normalise $S$ ), and so by Lemma $3.1,(x y)^{3}=1$. Therefore, by Theorem 3.15 and Corollary 3.14, $H^{\prime}$ acts 3-transitively on $\mathcal{O}$ and $H_{X}^{\prime}=\Gamma$. So $H^{\prime}$ is sharply 3-transitive on $\mathcal{O}$. The result then follows from Theorem 3.16.

Acknowledgements. We thank the referee for a careful reading of the paper and for their valuable comments.

\section{References}

[1] O. Amici and B. Casciaro. Concerning Buekenhout's theorem on Pascalian ovals. In Combinatorics '81 (Rome, 1981), volume 18 of Ann. Discrete Math., pages 17-27. North-Holland, Amsterdam-New York, 1983.

[2] R. Artzy. Pascal's theorem on an oval. Amer. Math. Monthly, 75:143-146, 1968.

[3] E. Beltrami. Saggio di interpretazione della Geometria non-euclidea. Giornale di Matematiche, 6:285$315,1868$.

[4] O. Bottema. The introduction of coordinates into projective geometry. Het invoeren van coördinaten in de projective meetkunde. Proc. Akad. Wet. Amsterdam, 31:1076-1085, 1928.

[5] F. Buekenhout. Études intrinsèque des ovales. Rend. Mat. e Appl. (5), 25:333-393, 1966.

[6] F. Buekenhout. Ovales et ovales projectifs. Atti Accad. Naz. Lincei Rend. Cl. Sci. Fis. Mat. Natur. (8), 40:46-49, 1966.

[7] F. Buekenhout. Plans projectifs à ovoïdes pascaliens. Arch. Math. (Basel), 17:89-93, 1966.

[8] G. Conti. Piani proiettivi dotati di un ovale pascaliano. Boll. Un. Mat. Ital. (4), 11(2):330-338, 1975.

[9] H. S. M. Coxeter. The Real Projective Plane. McGraw-Hill Book Company, Inc., New York, N. Y., 1949.

[10] T. De Medts and Y. Segev. A course on Moufang sets. Innov. Incidence Geom., 9:79-122, 2009.

[11] T. De Medts and R. M. Weiss. Moufang sets and Jordan division algebras. Math. Ann., 335(2):415433, 2006.

[12] G. Faina. An extension to abstract ovals of the Buekenhout theorem on Pascal ovals. Boll. Un. Mat. Ital. Suppl., 2:355-364, 1980.

[13] G. Faina and G. Korchmáros. The subgroup generated by regular involutions of a doubly transitive B-oval. Rend. Sem. Mat. Univ. Padova, 74:139-145, 1985.

[14] O. Fernandes. On an oval with the four point Pascalian property. Canad. Math. Bull., 27(3):295-300, 1984.

[15] O. Fernandes. On Pascal ovals. J. Austral. Math. Soc. Ser. A, 54(1):61-69, 1993.

[16] C. Hering, W. M. Kantor, and G. M. Seitz. Finite groups with a split $B N$-pair of rank 1. I. J. Algebra, 20:435-475, 1972.

[17] D. Hilbert. Neue Begründung der Bolyai-Lobatschefskyschen Geometrie. Math. Ann., 57(2):137-150, 1903.

[18] C. E. Hofmann, III. Specializations of Pascal's theorem on an oval. J. Geometry, 1:143-153, 1971.

[19] H. Karzel and K. Sörensen. Die lokalen Sätze von Pappus und Pascal. Mitt. Math. Gesellsch. Hamburg, 10(1):28-55, 1971.

[20] H. Karzel and K. Sörensen. Projektive Ebenen mit einem pascalschen Oval. Abh. Math. Sem. Univ. Hamburg, 36:123-125, 1971. Collection of articles dedicated to Lothar Collatz on his sixtieth birthday. 
[21] J. Kepler. Optics. Green Lion Press, Santa Fe, NM, 2000. Paralipomena to Witelo \& optical part of astronomy, Translated from the 1604 German original and with an introduction by William H. Donahue, With a preface by Dana Densmore and Donahue.

[22] W. Kerby and H. Wefelscheid. Conditions of finiteness on sharply 2-transitive groups. Aequationes Math., 8:287-290, 1972.

[23] G. Korchmáros. Su una classificazione delle ovali dotate di automorfismi. Rend. Accad. Naz. XL (5), 1/2:77-86 (1977), 1975/76.

[24] G. Korchmáros. On translation ovals in a Galois plane of even order. Rend. Accad. Naz. XL (5), 3:55-65 (1979), 1977/78.

[25] G. Korchmáros. Collineation groups transitive on the points of an oval $[(q+2)$-arc $]$ of $S_{2, q}$ for $q$ even. Atti Sem. Mat. Fis. Univ. Modena, 27(1):89-105 (1979), 1978.

[26] G. Korchmáros. A generalization of F. Buekenhout's theorem on Pascalian ovals. Boll. Un. Mat. Ital. B (5), 18(2):673-687, 1981.

[27] G. Korchmáros. 2-transitive abstract ovals of odd order. Aequationes Math., 33(2-3):208-219, 1987.

[28] W. A. Manning. Primitive groups. Part I. (Stanford University Publications. Univ. Series. Mathematics and Astronomy. Vol. 1 Nr. 1.). Stanford University, 108 S. gr. $8^{\circ}$ (1921)., 1921.

[29] G. E. Martin. Oval coordinates in a projective plane. In Atti del Convegno di Geometria Combinatoria e sue Applicazioni (Univ. Perugia, Perugia, 1970), pages 323-330. Ist. Mat., Univ. Perugia, Perugia, 1971.

[30] V. D. Mazurov. On sharply 2-transitive groups [translation of proceedings of the institute of mathematics, 30 (Russian), 114-118, Izdat. Ross. Akad. Nauk, Sibirsk. Otdel., Inst. Mat., Novosibirsk, 1996]. Siberian Adv. Math., 7(3):91-97, 1997. Siberian Advances in Mathematics.

[31] A. F. Möbius. Theorie der Kreisverwandschaft in rein geometrischer Darstellung. Ber. Verh. Sächs. Leipzig, 1855.

[32] J.-V. Poncelet. Traité des propriétés projectives des figures. Paris Gauthier-Villars, 1822.

[33] B. Riemann. Collected papers. Translated from the 1892 German edition by Roger Baker, Charles Christenson and Henry Orde. Heber City, UT: Kendrick Press, 2004.

[34] J. F. Rigby. Pascal ovals in projective planes. Canad. J. Math., 21:1462-1476, 1969.

[35] W. R. Scott. Group theory. Dover Publications, Inc., New York, second edition, 1987.

[36] Y. Segev. Proper Moufang sets with abelian root groups are special. J. Amer. Math. Soc., 22(3):889908, 2009.

[37] J. Tits. Généralisations des groupes projectifs basées sur leurs propriétés de transitivité. Acad. Roy. Belgique. Cl. Sci. Mém. Coll. in 8, 27(2):115, 1952.

[38] J. Tits. Twin buildings and groups of Kac-Moody type. In Groups, combinatorics E geometry (Durham, 1990), volume 165 of London Math. Soc. Lecture Note Ser., pages 249-286. Cambridge Univ. Press, Cambridge, 1992.

[39] O. Veblen and J. W. Young. Projective geometry. Vol. 1. Blaisdell Publishing Co. Ginn and Co. New York-Toronto-London, 1965.

(Bamberg) Centre for the Mathematics of Symmetry and Computation, School of Mathematics and Statistics, The University of Western Australia, 35 Stirling Highway, Crawley, W.A. 6009, Australia.

E-mail address: John.Bamberg@uwa.edu.au

(Harris) Centre for the Mathematics of Symmetry and Computation, School of Mathematics and Statistics, The University of Western Australia, 35 Stirling Highway, CrawLEY, W.A. 6009, Australia.

E-mail address: 21491993@student.uwa.edu.au

(Penttila) School of Mathematical Sciences, University of Adelaide, Adelaide, S.A. 5005, Australia. 